\title{
Insulin aggregation tracked by its intrinsic TRES
}

Li Hung C. Chung, David J. S. Birch, Vladislav Vyshemirsky, Maxim G. Ryadnov, and Olaf J. Rolinski

Citation: Appl. Phys. Lett. 111, 263701 (2017);

View online: https://doi.org/10.1063/1.5008477

View Table of Contents: http://aip.scitation.org/toc/apl/111/26

Published by the American Institute of Physics

\section{Articles you may be interested in}

Bidirectional reconfiguration and thermal tuning of microcantilever metamaterial device operating from $77 \mathrm{~K}$ to $400 \mathrm{~K}$

Applied Physics Letters 111, 261101 (2017); 10.1063/1.5006836

Experimental examination of tunneling paths in SiGe/Si gate-normal tunneling field-effect transistors Applied Physics Letters 111, 263504 (2017); 10.1063/1.4996109

Comparison of nanoparticle diffusion using fluorescence correlation spectroscopy and differential dynamic microscopy within concentrated polymer solutions

Applied Physics Letters 111, 263703 (2017); 10.1063/1.5016062

Evolution of a liquid-like fluid phase on $\mathrm{Ge} / \mathrm{Au}(111)$ at room temperature: A direct observation by STM Applied Physics Letters 111, 261601 (2017); 10.1063/1.5006684

Broadband manipulation of refracted wavefronts by gradient acoustic metasurface with V-shape structure Applied Physics Letters 111, 263501 (2017); 10.1063/1.5005950

Observing non-equilibrium state of transport through graphene channel at the nano-second time-scale Applied Physics Letters 111, 263101 (2017); 10.1063/1.5006258

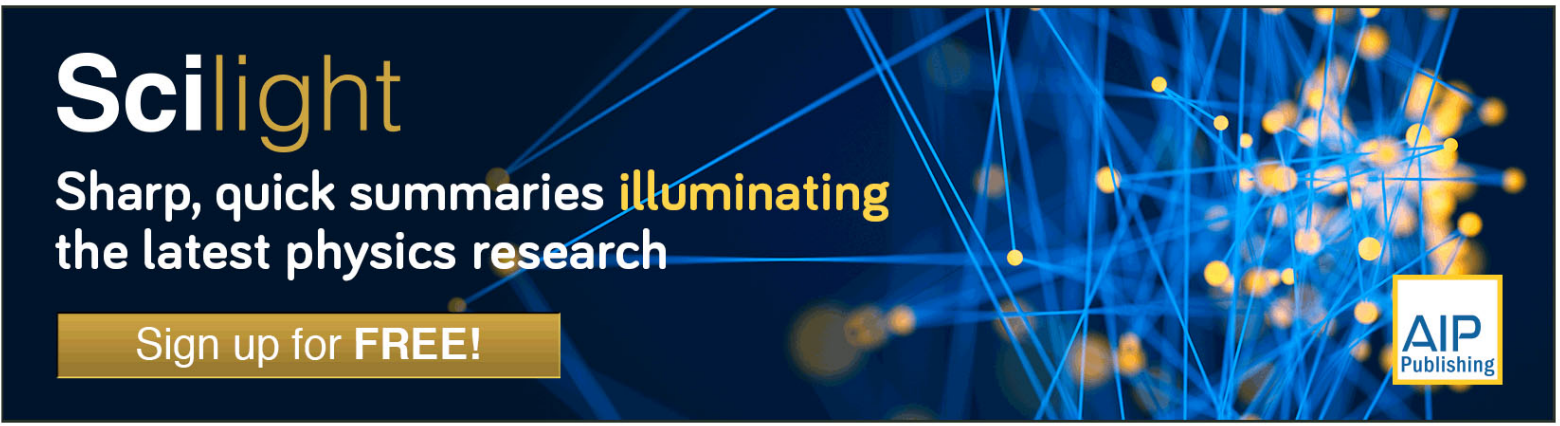




\title{
Insulin aggregation tracked by its intrinsic TRES
}

\author{
Li Hung C. Chung, ${ }^{1}$ David J. S. Birch, ${ }^{1}$ Vladislav Vyshemirsky, ${ }^{2}$ Maxim G. Ryadnov, ${ }^{3}$ \\ and Olaf J. Rolinski ${ }^{1, a)}$ \\ ${ }^{1}$ Photophysics Group, Centre for Molecular Nanometrology, Department of Physics, Scottish Universities \\ Physics Alliance, University of Strathclyde, 107 Rottenrow, Glasgow G4 0NG, United Kingdom \\ ${ }^{2}$ School of Mathematics and Statistics, University of Glasgow, Glasgow G12 8QQ, United Kingdom \\ ${ }^{3}$ National Physical Laboratory, Hampton Road, Teddington TW11 OLW, United Kingdom
}

(Received 6 October 2017; accepted 8 December 2017; published online 27 December 2017)

\begin{abstract}
Time-resolved emission spectra (TRES) have been used to detect conformational changes of intrinsic tyrosines within bovine insulin at a physiological $\mathrm{pH}$. The approach offers the ability to detect the initial stages of insulin aggregation at the molecular level. The data analysis has revealed the existence of at least three fluorescent species undergoing dielectric relaxation and significant spectral changes due to insulin aggregation. The results indicate the suitability of the intrinsic TRES approach for insulin studies and for monitoring its stability during storage and aggregation in insulin delivery devices. Published by AIP Publishing. https://doi.org/10.1063/1.5008477
\end{abstract}

Protein aggregation has attracted significant interest due to its role in neurodegenerative diseases including Alzheimer's disease (AD), Parkinson's disease (PD), and diabetes type II. ${ }^{1,2}$ Proteins and their proteolytic products can undergo conformational changes allowing them to selfassemble into nanostructured amyloid fibrils. Such structures then accumulate into microscopic deposits, senile plaques, which are found in the post-mortem brains of AD patients. However, correlations between the amyloid fibrils and the extent of cognitive loss have yet to be reported. By contrast, cytotoxicity is attributed to the soluble, prefibrillar forms of amyloid ${ }^{3}$ whereas reducing fibril formation does not necessarily lower cytotoxicity. ${ }^{4}$ Taken together, these factors suggest that insoluble amyloid fibrils are the repositories of soluble intermediates that remain in equilibrium with insoluble fibrillar forms. ${ }^{5}$ Therefore, it is believed that the fibriltype of protein aggregation should follow three main steps: (1) misfolding of the monomeric form into a low oligomer, which (2) nucleates the formation of elongated proto-fibrils that (3) aggregate forming mature fibrils. ${ }^{6,7}$ Most importantly, proteopathies-neurodegenerative disorders caused by protein misfolding and aggregation-are caused by different proteins, ${ }^{6}$ but all result from the same generic mechanism of fibrillogenesis. Yet, a capability to monitor this very process of persistent protein aggregation is lacking.

One protein that is prone to fibrillation is insulin-a hormone which regulates blood glucose levels within the body. Patients with diabetes do not produce enough insulin to stabilize blood glucose levels and thus need additional injections of this hormone. Insulin is an extensively studied protein because of complications caused by its aggregation. For example, at frequent injection sites, long fibrous insulin structures have been found. ${ }^{8-10}$ Also, the long term storage of insulin has been shown to lead to aggregation, thus impacting the efficacy and cost of diabetes treatment. ${ }^{11}$ Specifically, the transportation and storage at higher temperatures increase the likelihood of instability since fibril and covalent polymer formation are temperature-dependent. The

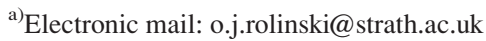

devices used in insulin infusion therapy are particularly susceptible because wearing at body heat, shaking of the reservoir with bubble formation, and interaction with hydrophobic surfaces of the infusion cannula all encourage monomer formation and partial monomer unfolding, the prerequisites for fibril formation. Moreover, serum samples taken from patients suffering from PD displayed an autoimmune response to insulin fibrils, which may suggest that insulin aggregation may play a role in neurodegenerative disease. ${ }^{12}$

Insulin $^{13}$ consists of 51 residues with two polypeptide chains, A and B. Chain A has 21 amino acid residues and a disulphide bridge between two cysteine amino acids, while chain B is composed of 30 amino acids. Both chains are covalently linked together by two cysteine bridges. There are seven intrinsic fluorophores in an insulin particle: two tyrosine (Tyr) residues on chain A and two Tyr and three phenylalanine (Phe) residues on chain B. Insulin is predominantly stored as a hexamer stabilized by zinc ions and favours hexamer conformations at $\mathrm{pH}$ 7.4.

Several techniques have been employed to study fibril formation including circular dichroism (CD), ${ }^{14,15}$ transmission electron microscopy (TEM), ${ }^{16}$ atomic force microscopy (AFM),${ }^{17} \mathrm{X}$-ray diffraction, ${ }^{18}$ and others. ${ }^{19-21}$

CD spectroscopy studies showed that insulin adopts $\alpha$ helical conformations that can convert to appreciably $\beta$ pleated structures as a function of time. X-ray diffraction studies showed structural similarities between insulin fibrils and other proteins associated with amyloid formation, in which individual $\beta$-strands run perpendicular to the main fibril axis. The cross- $\beta$ fibril structure is the hallmark of amyloid fibrils.

Fluorescence spectroscopy is one of the most informative techniques to study protein folding and self-assembly and to monitor conformational changes. It offers an excellent combination of speed, ease of use, and nanometre-level molecular information. ${ }^{22}$ Fluorescence benefits from labelling the protein assembly of interest with extrinsic fluorophores such as Thioflavin T (ThT), Auramine O, ANS, and Congo red ${ }^{13,23-25}$ to probe secondary structure alterations as a function of fluorescence responses. The use of these dyes 
has given a deeper understanding of protein fibrillation. The approaches used include conventional fluorescence spectra, ${ }^{26}$ fluorescence decay, ${ }^{27}$ and fluorescence correlation spectroscopy. ${ }^{28}$ One of the main findings observed in ThT-based experiments was ThT fluorescence intensity showing a sigmoidal function with negligible fluorescence intensity observed in the early stages, a gradual increase over time, and a plateau in the final stage. As the ThT quantum yield increases from nearly 0 to a higher value on binding to the $\beta$ sheets of probed fibrils, this result indicates that fibril formation by insulin undergoes three stages: an initial lag phase, a growth phase, and a final equilibrium phase.

However, the use of extrinsic fluorophores is associated with some limitations as they can perturb protein kinetics, which leads to ambiguities in interpretation and lacks the sensitivity required for studying prefibrillar structures, ${ }^{29}$ and different probes used on the same system can yield different signals. ${ }^{30}$ In this study, we attempt to overcome these limitations with the use of intrinsic fluorophores to detect conformational changes during insulin fibrillation. Our premise is that since insulin has four tyrosine residues which are sensitive to the local microenvironments, their fluorescence response may carry information relevant to the insulin aggregation and fibrillation. In previous studies of tyrosine response to insulin fibrillation, e.g. ${ }^{13}$ its fluorescence was monitored at the fixed wavelength only and insulin fibril formation was accelerated by elevating temperatures and/or decrease $\mathrm{pH}$ of the aqueous solution, which is inconsistent with physiological conditions.

In this paper, we report insulin's tyrosine fluorescence intensity decay and time-resolved emission spectra (TRES) to extract in-depth information on insulin aggregation kinetics under physiological $\mathrm{pH}$ conditions. Using time-resolved techniques carries significant advantages over the timeintegrated approaches, ${ }^{22}$ the latter offering limited information on the dynamics of molecules in the excited-states.

Bovine insulin and phosphate-buffered saline $\mathrm{pH} 7.4$ (PBS) were purchased from Sigma-Aldrich and were used without further purification. $50 \mu \mathrm{M}$ solution of bovine insulin was prepared in PBS buffer $(0.01 \mathrm{M})$ and sonicated until completely dissolved. The sample was kept and measured at $22{ }^{\circ} \mathrm{C}$. The steady state absorption and fluorescence spectra measurements were recorded using a Lambda $25 \mathrm{UV}$-Vis spectrometer (Perkin-Elmer) and Fluorolog (Horiba Scientific), respectively. For TRES measurements, the fluorescence decays were recorded on a DeltaFlex fluorescence lifetime system (Horiba Jobin Yvon IBH Ltd, Glasgow) using time correlated single photon counting (TCSPC). A NanoLED producing $\sim 600$ ps pulses at $279 \mathrm{~nm}^{31}$ was used to excite the intrinsic tyrosine fluorescence at detection wavelengths $\lambda$ ranging from 290 to $350 \mathrm{~nm}$ at $5 \mathrm{~nm}$ intervals. To avoid the influence of rotational effects on fluorescence decays, a vertically oriented polariser in the excitation channel and a polarizer at the magic angle $\left(54.7^{\circ}\right)$ in the detection channel were added in the optical path. Fluorescence decays recorded at the series of $\lambda$ values were fitted to a multi-exponential model function

$$
I_{\lambda}(t)=\sum_{i=1}^{N} \alpha_{i}(\lambda) \exp \left(\frac{-t}{\tau_{i}(\lambda)}\right)
$$

where $\alpha_{i}(\lambda)$ is the $i$-th pre-exponential component and $\tau_{i}(\lambda)$ is the relevant fluorescence lifetime. The fitting procedure applied here assumed that the experimental decay curve is the combination of the fluorescence decay function (1), the background signal, and the contribution of the scattered excitation light as described previously. ${ }^{32}$ The nonlinear least squares approach using $\chi^{2}$ and weighted residuals as the goodness of fit criteria has shown that a 3 -exponential model $(N=3)$ is sufficient to provide an accurate representation of the fluorescence decays. The recovered parameters $\alpha_{i}(\lambda)$ and $\tau_{i}(\lambda)$ were then used to create TRES. The time resolved emission spectra $I_{t}(\lambda)$ were obtained according to the following equation: $:^{33,34}$

$$
I_{t}(\lambda)=\sum_{i=1}^{3} \frac{\alpha_{i}(\lambda) \exp \left(\frac{-t}{\tau_{i}(\lambda)}\right) S(\lambda)}{\sum_{i} \alpha_{i}(\lambda) \tau_{i}},
$$

where $S(\lambda)$ is the steady-state spectrum of the sample. Each $S(\lambda)$ spectrum has been corrected for a dark signal offset, fluctuations in the lamp output, and the wavelength response of the monochromator's diffraction grating. In order to obtain TRES as a function of wavenumber, the $I_{t}(\lambda)$ were converted to $I_{t}(\nu)$ by $^{35,36}$

$$
I_{t}(\nu)=\lambda^{2} I_{t}(\lambda) .
$$

In the next stage, the model of TRES $g_{t}(\nu)$, based on the Toptygin-type approach ${ }^{37}$ where the multiple normalised Gaussian distributions were used to represent the spectral shapes of fluorescent components

$$
g_{t}(\nu)=\nu^{3} \sum_{i}^{3} \frac{A_{i}(t)}{\sqrt{2 \pi \sigma_{i}(t)^{2}}} \exp \left(\frac{-\left(\nu-\nu_{i}(t)\right)^{2}}{2 \sigma_{i}(t)^{2}}\right),
$$

was fitted to the experimental spectra (3). Here, $A_{i}(t)$ is the contribution of each component, $\nu_{i}(\mathrm{t})$ is the peak position, and $\sigma_{i}(\mathrm{t})$ is the half-width of the distribution. In practice, a sum of 3 Gaussian distributions was fitted to the expression $\nu^{-3} I_{t}(\nu)$.

Fluorescence spectra of insulin at different times of aggregation are shown in Fig. 1. The results cover the period from about $20 \mathrm{~min}$ after sample preparation up to over $600 \mathrm{~h}$. The intensity of the initial peak at about $300 \mathrm{~nm}$ gradually decreases, and the spectrum becomes broader and shifts towards longer wavelengths. Normalised spectra [Fig. 1(B)] show at least three characteristic peak values about $300 \mathrm{~nm}$, $315 \mathrm{~nm}$, and $340 \mathrm{~nm}$, indicating fluorescence from the products of the complex conformational changes, which cannot be explained on the basis of the steady-state studies only. The drop in Tyr fluorescence intensity is consistent with the result reported by Bekard and Dunstan, ${ }^{13}$ except that in the conditions of their experiment, $\mathrm{pH} 1.9$ and temperature $60^{\circ} \mathrm{C}$, the aggregation occurred within $10 \mathrm{~h}$, while in our experiment, $\mathrm{pH} 7.4$ and $22^{\circ} \mathrm{C}$, it takes about 30 days. This result confirms that at physiological $\mathrm{pH}$ and in the presence of Zinc ions, the insulin hexamer formation is stable, which is exploited for stabilisation of insulin in neutral $\mathrm{pH}$ drug product formulations. 

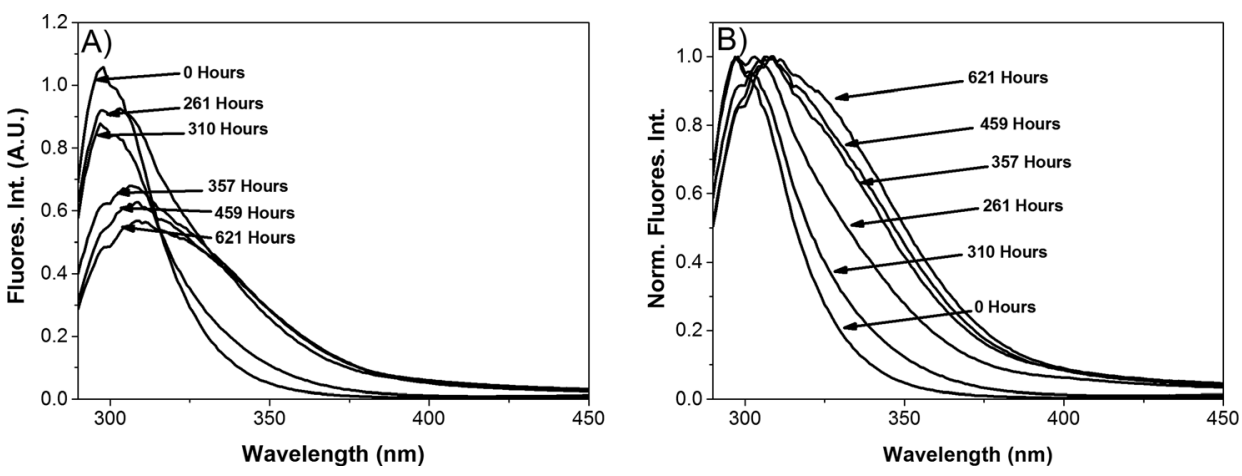

FIG. 1. Fluorescence spectra of $50 \mu \mathrm{M}$ insulin in PBS buffer at selected times of $0,261,310,357,459$, and $621 \mathrm{~h}$ after sample preparation (A) and the normalised spectra (B).
The fluorescence intensity decays of the insulin sample were recorded at different wavelengths and at different points of aggregation. Figure S1 in the supplementary material demonstrates strong lifetime-wavelength dependence where the fluorescence intensity decays measured at the short wavelength side of the spectrum $(290 \mathrm{~nm})$ decay more rapidly than the decays measured at the longer wavelength side $(350 \mathrm{~nm})$. This is consistent with the lifetime-wavelength correlation observed in proteins. ${ }^{38,39}$ The effect may be explained by the fluorescence intensity decay measured at the short-wavelength side of the spectrum being accelerated by the peak of the transient emission spectrum shifting away from the detection wavelength due to dielectric relaxation, thus causing a further decrease in intensity. For the same reason, the decay detected at the long-wavelength side of the spectrum decreases at a slower rate, as the peak of the shifting transient emission spectrum moves towards the detection wavelength. However, the detailed analysis of the recovered fluorescence lifetimes $\tau_{i}(\lambda)$ [see Table S1 and Fig. S1(C) in the supplementary material] shows that although the non-significant increase in two shorter lifetimes $\tau_{i}(\lambda)$ is typical for the lifetime-wavelength dependence, the strong increase in the longest lifetime has to be a consequence of significant structural change. The comparison of Figs. S1(A) and S1(B) and the differences in the $\tau_{i}(\lambda)$ in Fig. S1(C) show that the lifetime components of insulin after $690 \mathrm{~h}$ of aggregation have increased as compared to the lifetimes observed at 0.35 hours $(21 \mathrm{~min})$. This is likely to be due to numerous effects related to the complexity of insulin photophysics. Indeed, insulin has four tyrosine residues and three of them are buried in the protein ${ }^{40}$ and therefore can be simultaneously involved in both hydrophobic and hydrophilic interactions. Therefore, the alterations in the local environments of the tyrosines at different stages of insulin aggregation are likely to result in more complex fluorescence kinetics. Nevertheless, because the fluorescence lifetime components $\tau_{i}(\lambda)$ are dependent on $\lambda$, the decay associated spectra (DAS) technique, assuming the spectrum to be the sum of the stationary spectra of few different forms, is not applicable and the TRES approach is needed.

TRES $I_{t}(\nu)$ were determined for the insulin sample at different times after its preparation. The examples of the $\nu^{-3} I_{t}(\nu)$ plots vs. time for the fresh $(0.35$ hours after preparation) sample and the old (690 $\mathrm{h}$ after preparation) sample are shown in Figs. 2(A) and 2(B), respectively. Both sets of plots $\nu^{-3} I_{t}(\nu)$ display peaks around $33000 \mathrm{~cm}^{-1}$ whose intensities gradually decrease with time after excitation. The main difference between both samples is the emergence of a broader structure at shorter wavenumbers observed in the old sample. This is better demonstrated in the normalised plots of Figs. 2(C) and 2(D) where it appears at $\sim 30000 \mathrm{~cm}^{-1}$.

To obtain fuller and quantitative information on the observed spectral changes, all $\nu^{-3} I_{t}(\nu)$ spectra were modelled
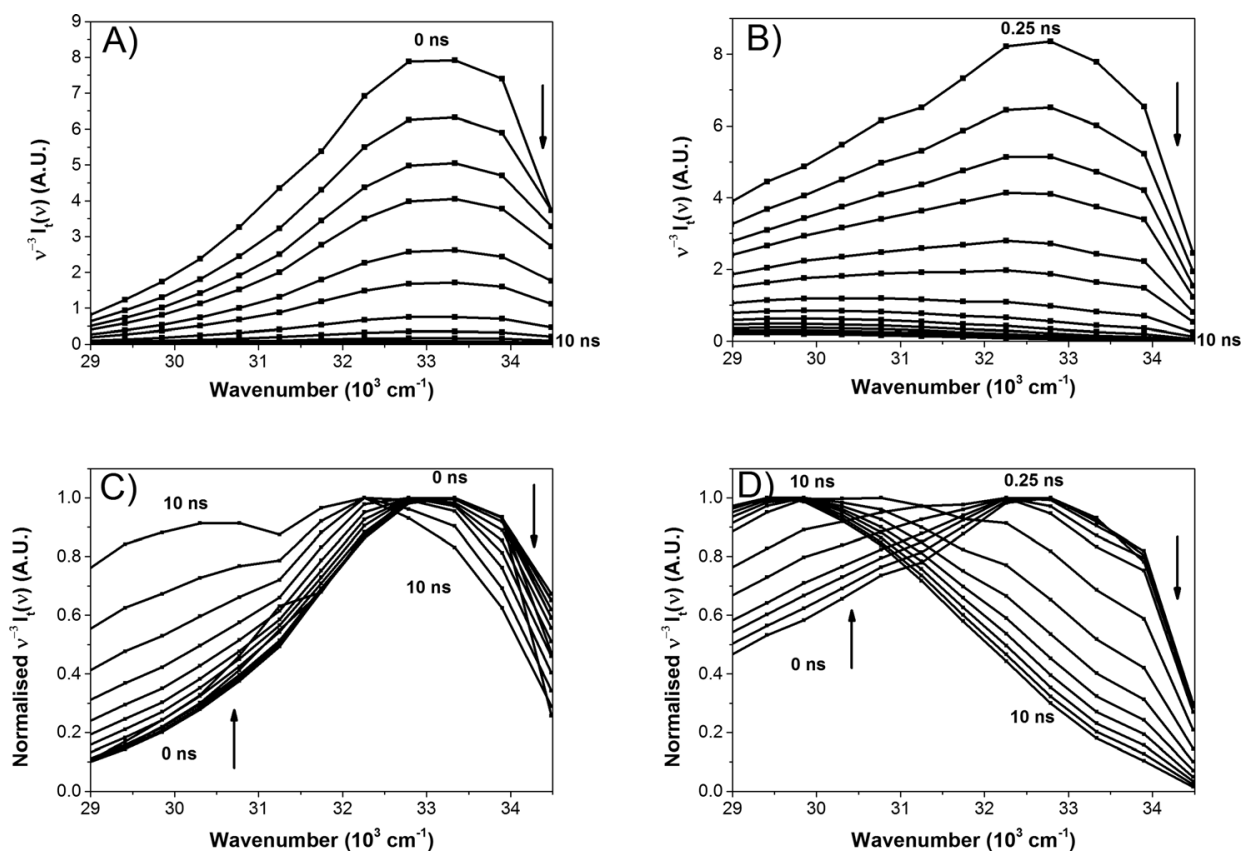

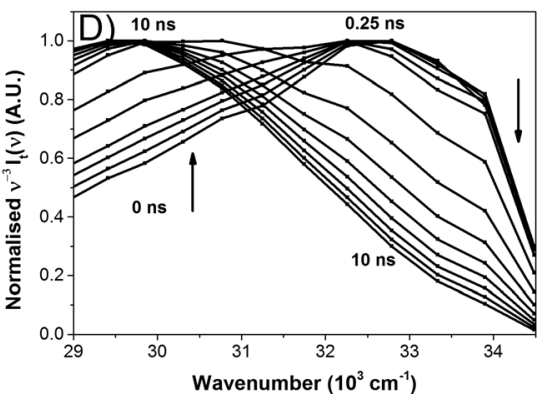

FIG. 2. TRES spectra of (A) 0.35 and (B) $690 \mathrm{~h}$ old insulin samples determined at $0,0.25,0.5,0.75,1,1.5,2,3$, $4,5,6,7,8,9$, and 10 ns after excitation. Plots (C) and (D) show the same spectra after normalisation. 
by the sum of 3 normalised Gaussian profiles (the sum of two Gaussians fitted poorly to the experimental data, while the $\chi^{2}$ values obtained for the triple-Gaussian fitting were in the acceptable range of 1.00-1.19. Figure S2 (supplementary material) shows the examples of fitting the triple-Gaussian function to the data obtained for the fresh and old samples (as in Fig. 2) for the times $0.25 \mathrm{~ns}$ and $10 \mathrm{~ns}$ after excitation. The fresh sample shows initially (at $0.25 \mathrm{~ns}$ after excitation) two emitting species with the maxima around 34000 and $33000 \mathrm{~cm}^{-1}$ (as recovered from Gaussian fitting) and, after $10 \mathrm{~ns}$, the additional component with the peak at $30000 \mathrm{~cm}^{-1}$. In the old sample, the initial spectrum shows three peaks: at 34000 and $33000 \mathrm{~cm}^{-1}$ as in the fresh sample and the dominating component with the peak at $31000 \mathrm{~cm}^{-1}$. After $10 \mathrm{~ns}$, this peak shifts to around $29500 \mathrm{~cm}^{-1}$.

The above observations seem to be consistent with compilation of two processes characteristic of proteins: ${ }^{21}$ excitationtriggered conformational change of insulin molecules on a nanosecond time scale and their aggregation in the time-scale of several hours to days.

As can be seen in Fig. 2(D), there is no gradual shift of the spectrum on the nanosecond scale, which would be consistent with the dielectric relaxation, but the spectrum is rather a sum of two profiles with the maxima at $33000 \mathrm{~cm}^{-1}$ and $30000 \mathrm{~cm}^{-1}$, which suggests that the $30000 \mathrm{~cm}^{-1}$ profile is the conformational form. On the other hand, the origin of the profile with the peak at $31000 \mathrm{~cm}^{-1}$ in the initial spectrum of the old sample [Fig. S2(C), supplementary material] can be explained as the result of aggregation of the molecules that emitted at $33000 \mathrm{~cm}^{-1}$ before their aggregation. This also explains the reduced intensity of the $33000 \mathrm{~cm}^{-1}$ band. The aggregates also seem to undergo a conformational change in a ns time scale, which causes further shift of their spectra maximum to about $29500 \mathrm{~cm}^{-1}$ as is seen in the old sample TRES at $10 \mathrm{~ns}$ [Fig. S2(D), supplementary material].

The peaks of the Gaussian profiles fitted to the experimental $\nu^{-3} I_{t}(\nu)$ curves are plotted in Fig. 3(A). The locations of peaks emitting around $34000 \mathrm{~cm}^{-1}$ and $33000 \mathrm{~cm}^{-1}$ seem to be weakly dependent on the age of the sample and show a non-significant spectral shift towards longer wavelengths which is consistent with the effect of dielectric relaxation. Indeed, contrary to most of the fluorophores for which dielectric relaxation is much faster than fluorescence decay, the dielectric relaxation of intrinsic fluorophores like tyrosine and tryptophan in proteins usually occurs on the same nanosecond time scale as fluorescence which leads to more complex photophysics.

The spectral shifts of the third component, however, are unlikely to be due the dielectric relaxation because their values are significant, some emission about $33000 \mathrm{~cm}^{-1}$ remains in the spectrum at later times, and the shift of the peak vs. time dependence does not always have a dielectric relaxation exponential character but a sigmoidal shape in some cases. Therefore, we currently interpret the spectral shifts of the third component as a result of the excitationtriggered conformational changes of the insulin aggregates. The variations in the third peak position vs. time plots observed at different ages of the sample may be explained by different compositions of the insulin aggregates during its aging.

Since our model of the insulin fluorescence spectra [Eq. (4)] uses the normalised Gaussian distribution functions, the amplitudes $A_{i}(t)$ represent the contributions of fluorescence from three components. Consequently, the time decays of $A_{i}(t)$ can be regarded as the fluorescence decay of these components. Figure 3(B) shows the $A_{i}(t)$ decays observed in the oldest sample. Exponential fitting of these curves gives the fluorescence lifetimes of the $34000 \mathrm{~cm}^{-1}$ and $33000 \mathrm{~cm}^{-1}$ forms as $0.96 \mathrm{~ns}$ and $1.10 \mathrm{~ns}$, respectively, while the fluorescence lifetime of the conformational form is longer$1.39 \mathrm{~ns}$. Note that these values are substantially shorter than the longest lifetime components recovered from the raw decays [Fig. S1(C) and Table S1 in the supplementary material], which shows how effects like dielectric relaxation and conformational change in the fluorophore may complicate interpretation of the lifetime data.

By using the TRES technique, we have provided a new insight into the tyrosine fluorescence kinetics of bovine insulin during its aggregation. We have observed the fluorescence of two forms showing peak emission at $34000 \mathrm{~cm}^{-1}$ and $33000 \mathrm{~cm}^{-1}$ and the fluorescence of the product of conformational change of the $33000 \mathrm{~cm}^{-1}$ form with the peak emission at $30000 \mathrm{~cm}^{-1}$. As the insulin solution ages, the aggregation of the $33000 \mathrm{~cm}^{-1}$ form occurs resulting in the shift of the initial aggregate emission peak to $31000 \mathrm{~cm}^{-1}$. The aggregates also undergo conformational change after excitation leading to the emission with the peak shifted to $\sim 29000 \mathrm{~cm}^{-1}$. Identification and further characterisation of
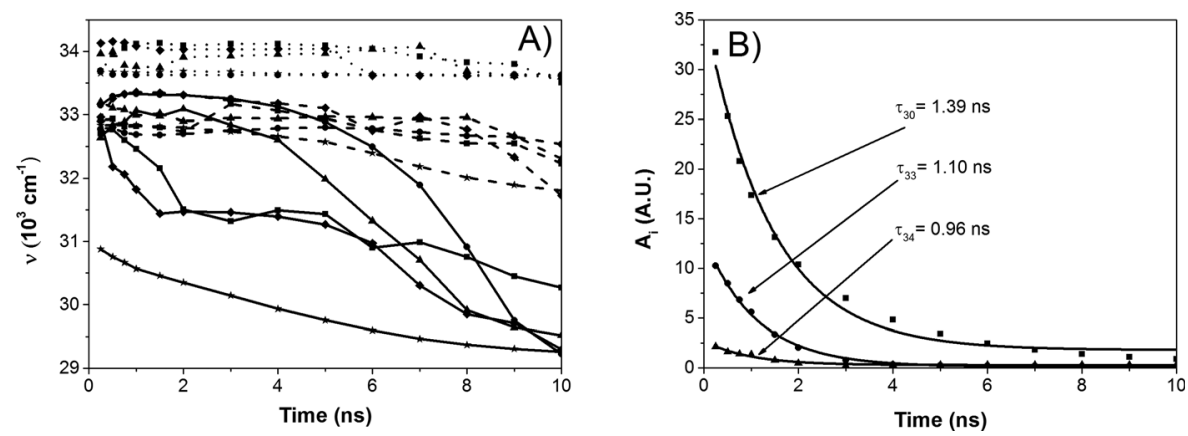

FIG. 3. (A) Time evolution of the peak positions of the fluorophores emitting at $34000 \mathrm{~cm}^{-1}$ (dotted line), $33000 \mathrm{~cm}^{-1}$ (dashed line), and $30000 \mathrm{~cm}^{-1}$ (solid line) after excitation for the sample measured after $0.35(\mathbf{\square}), 22(\bullet), 68(\boldsymbol{\Delta}), 93(\bullet)$, and $690(\boldsymbol{\star}) \mathrm{h}$ of aggregation. Plot (B) shows time evolution of the contributions of Gaussian components $A_{i}(t)$ in the oldest sample. Shown are the forms with the initial peak at $34000 \mathrm{~cm}^{-1}(\mathbf{\Lambda}), 33000 \mathrm{~cm}^{-1}(\mathbf{O})$, and $30000 \mathrm{~cm}^{-1}(\mathbf{\square})$. 
the fluorescent insulin forms will require further TRES studies for the samples at different concentrations, temperatures, and $\mathrm{pH}$ conditions. Nevertheless, we have demonstrated that tracking the changes in the intrinsic fluorescence offers a sensitive way of studying unfolding and aggregation of insulin when not modified by extrinsic markers and in the undisturbed clinically relevant environment, for example, inside a plastic delivery cannula or within a storage vial.

See supplementary material for the fluorescence intensity decays of the fresh and older insulin samples (detected at different emission wavelengths) and the changes in the recovered lifetimes (Fig. S1, supplementary material), examples of fitting the model of TRES [Eq. (4)] to the actual spectra (Fig. S2, supplementary material), and the results of fitting the fluorescence intensity decays $I_{\lambda}(t)$ of the insulin sample recorded at different wavelengths and at different points of aggregation (Table S1, supplementary material).

The authors thank Professor John Pickup (Guy's Hospital, London) for his valuable discussion on the importance of insulin stability. This work was supported in part by the UK's Department for Business, Energy and Industrial Strategy.

${ }^{1}$ T. P. J. Knowles, M. Vendruscolo, and C. M. Dobson, Nat. Rev. Mol. Cell Biol. 15, 384 (2014).

${ }^{2}$ D. Eisenberg and M. Jucker, Cell 148, 1188 (2012).

${ }^{3}$ M. Sakono and T. Zako, FEBS J. 277, 1348 (2010).

${ }^{4}$ L. Jiang, C. Liu, D. Leibly, M. Landau, M. Zhao, M. P. Hughes, and D. S. Eisenberg, Elife 2013, e00857.

${ }^{5}$ J. Bieschke, M. Herbst, T. Wiglenda, R. P. Friedrich, A. Boeddrich, F. Schiele, D. Kleckers, J. M. Lopez del Amo, B. A. Grüning, Q. Wang, M. R. Schmidt, R. Lurz, R. Anwyl, S. Schnoegl, M. Fändrich, R. F. Frank, B. Reif, S. Günther, D. M. Walsh, and E. E. Wanker, Nat. Chem. Biol. 8, 93 (2012).

${ }^{6}$ F. Chiti and C. M. Dobson, Annu. Rev. Biochem. 75, 333 (2006).

${ }^{7}$ L. Nielsen, R. Khurana, A. Coats, S. Frokjaer, J. Brange, S. Vyas, V. N. Uversky, and A. L. Fink, Biochemistry 40, 6036 (2001).

${ }^{8}$ F. E. Dische, C. Wernstedt, G. T. Westermark, P. Westermark, M. B. Pepys, J. A. Rennie, S. G. Gilbey, and P. J. Watkins, Diabetologia 31, 158 (1988).

${ }^{9}$ J. Brange, L. Andersen, E. D. Laursen, G. Meyn, and E. Rasmussen, J. Pharm. Sci. 86, 517 (1997)

${ }^{10}$ S. Störkel, H. M. Schneider, H. Müntefering, and S. Kashiwagi, Lab Invest. 48, 108 (1983).

${ }^{11}$ V. Sluzky, J. A. Tamada, A. M. Klibanov, and R. Langer, Proc. Natl. Acad. Sci. 88, 9377 (1991).
${ }^{12}$ K. R. Wilhelm, K. Yanamandra, M. A. Gruden, V. Zamotin, M. Malisauskas, V. Casaite, A. Darinskas, L. Forsgren, and L. A. MorozovaRoche, Eur. J. Neurol. 14, 327 (2007).

${ }^{13}$ I. B. Bekard and D. E. Dunstan, Biophys. J. 97, 2521 (2009).

${ }^{14}$ Q. X. Hua and M. A. Weiss, J. Biol. Chem. 279, 21449 (2004).

${ }^{15}$ A. Ahmad, V. N. Uversky, D. Hong, and A. L. Fink, J. Biol. Chem. 280, 42669 (2005).

${ }^{16}$ T. Zako, M. Sakono, N. Hashimoto, M. Ihara, and M. Maeda, Biophys. J. 96, 3331 (2009).

${ }^{17}$ E. Kachooei, A. A. Moosavi-Movahedi, F. Khodagholi, H. Ramshini, F. Shaerzadeh, and N. Sheibani, PLoS One 7, e41344 (2012).

${ }^{18}$ M. Sunde, L. C. Serpell, M. Bartlam, P. E. Fraser, M. B. Pepys, and C. C. F. Blake, J. Mol. Biol. 273, 729 (1997).

${ }^{19}$ M. Bouchard, J. Zurdo, E. J. Nettleton, C. M. Dobson, and C. V. Robinson, Protein Sci. 9, 1960 (2000).

${ }^{20}$ A. Gladytz, E. Lugovoy, A. Charvat, T. Häupl, K. R. Siefermann, and B. Abel, Phys. Chem. Chem. Phys. 17, 918 (2015).

${ }^{21}$ E. J. Nettleton, P. Tito, M. Sunde, M. Bouchard, C. M. Dobson, and C. V. Robinson, Biophys. J. 79, 1053 (2000).

${ }^{22}$ J. R. Lakowicz, Principles of Fluorescence Spectroscopy, 3rd ed., edited by J. R. Lakowicz (Springer, 2006).

${ }^{23}$ M. R. Nilsson, Methods 34, 151 (2004).

${ }^{24}$ R. Khurana, C. Coleman, C. Ionescu-Zanetti, S. A. Carter, V. Krishna, R. K. Grover, R. Roy, and S. Singh, J. Struct. Biol. 151, 229 (2005).

${ }^{25}$ N. H. Mudliar, A. M. Pettiwala, A. A. Awasthi, and P. K. Singh, J. Phys. Chem. B 120, 12474 (2016).

${ }^{26}$ Y. Hong, L. Meng, S. Chen, C. W. T. Leung, L. T. Da, M. Faisal, D. A. Silva, J. Liu, J. W. Y. Lam, X. Huang, and B. Z. Tang, J. Am. Chem. Soc. 134, 1680 (2012).

${ }^{27}$ J. Mohanty, S. D. Choudhury, H. Pal, and A. C. Bhasikuttan, Chem. Commun. 48, 2403 (2012).

${ }^{28}$ Z.-H. Zhong, A. Pramanik, K. Ekberg, O. T. Jansson, H. Jörnvall, J. Wahren, and R. Rigler, Daibetologia 44, 1184 (2001).

${ }^{29}$ Y. Porat, A. Abramowitz, and E. Gazit, Chem. Biol. Drug Des. 67, 27 (2006).

${ }^{30}$ A. J. Thompson, T. W. Herling, M. Kubánková, A. Vyšniauskas, T. P. J. Knowles, and M. K. Kuimova, J. Phys. Chem. B 119, 10170 (2015).

${ }^{31}$ C. D. McGuiness, K. Sagoo, D. McLoskey, and D. J. S. Birch, Meas. Sci. Technol. 15, L19 (2004).

${ }^{32}$ O. J. Rolinski, T. Wellbrock, D. J. S. Birch, and V. Vyshemirsky, J. Phys. Chem. Lett. 6, 3116 (2015).

${ }^{33}$ J. R. Lakowicz, E. Gratton, H. Cherek, B. P. Maliwal, and G. Laczko, J. Biol. Chem. 259, 967 (1984).

${ }^{34}$ M. Badea and L. Brand, Methods Enzymol. 61, 378 (1979).

${ }^{35}$ R. F. Chen, Anal. Biochem. 19, 374 (1967).

${ }^{36}$ B. Valeur and M. N. Berberan-Santos, Molecular Fluorescence: Principles and Applications, 3rd ed. (Wiley VCH, Weinheim, 2013).

${ }^{37}$ D. Toptygin and L. Brand, Chem. Phys. Lett. 322, 496 (2000).

${ }^{38}$ O. J. Rolinski, D. McLaughlin, D. J. S. Birch, and V. Vyshemirsky, Methods Appl. Fluoresc. 4, 24001 (2016).

${ }^{39}$ J. R. Lakowicz, Photochem. Photobiol. 72, 421 (2000).

${ }^{40}$ E. H. Strickland and D. Mercola, Biochemistry 15, 3875 (1976). 